\title{
Sensitivity of the phenoxy derivatives of 2,4-dihydro- $5 H$-[1,2,3]triazolo[4,5-d]pyrimidin-5-ones to acidic and basic stimuli
}

\author{
Alexander K. Eltyshev (1), Nataliya P. Belskaya* (10) \\ Ural Federal University, 19 Mira St., Yekaterinburg 620002, Russia \\ * Corresponding author: n.p.belskaya@urfu.ru \\ This article belongs to the regular issue. \\ (C) 2021, The Authors. This article is published in open access form under the terms and conditions of the Creative \\ Commons Attribution (CC BY) license (http://creativecommons.org/licenses/by/4.0/).
}

\section{Abstract}

Herein we report the research on the sensitivity of six hydroxy derivatives of dihydrotriazolopyrimidines (HO-DTP) to acids and bases. The UV/Vis and fluorescence spectra of these compounds were investigated with the addition of the acids and bases. Spectral data revealed the strong red shifts for emission and absorption maxima in the presence of $\mathrm{KOH}$ and $\mathrm{NaOH}$. Moreover, two DTPs demonstrated strengthening of the emission intensity. The obtained results and data published in our previous paper demonstrated the strong and selective sensory response of DTPs to the acids and bases and elucidated relationships between the structure and sensitivity to the environment. This finding allowed us to manage these properties by introducing the combination of substituents and functionalities into the heterocyclic core. Thus, investigations demonstrated the potential of the application of DTPs as chemo- and fluorosensors for selective detection of acids and bases.

\section{Keywords}

dihydrotriazolopyrimidines

acids

bases

fluorescence response

chemosensor

Received: 27.07.2021

Revised: 11.08.2021

Accepted: 16.08.2021

Available online: 17.08 .2021

\section{Introduction}

Fluorophores sensitive to external stimuli are attractive compounds owing to their application in contaminant analysis environment, electrochemical sensors, biosensing, and detection of toxins [1]. Extensive studies have been directed for development of new fluorescent sensory organic materials which are able to respond on the external stimuli via changes of their absorption or fluorescence characteristics. These stimuli may include temperature, scattered light, $\mathrm{pH}$, and even nature of solvent [2].

Monitoring the $\mathrm{pH}$ levels in high alkaline media is of wide importance for many industrial processes, in gas scrubbers, wastewater monitoring, treatment of the plants and concrete structures [3, 4]. The sensing materials suitable for measurement of high alkali media are very limited. In recent years, a few fluorescent sensors in an alkaline $\mathrm{pH}$ region have been reported [5-7].

Recently we reported the synthesis and photophysical properties of new blue and blue-green fluorophores derived on dihydro-1,2,3-triazolopyrimidine core (DTP) [8]. These fluorophores exhibited multifunctional properties and showed good fluorescence in solutions (with QY up to $88 \%$, Stokes shift (SS) $\sim 151 \mathrm{~nm}$ and $\lambda_{\text {em }}$ up to $534 \mathrm{~nm}$ ). We suggested and developed synthetic procedure providing various DTPs with different electronic structures (A- $\pi-\mathrm{D}$ and $D-\pi-A$ ). These compounds showed selective reversible acidochromism in solution and in a solid state in the presence of $\mathrm{HCl}$ vapor. ${ }^{1} \mathrm{H}$ NMR investigation showed that the pyrimidine and $N$-tert-cycloalkylamine moieties are the binding sites responsible for specific sensory response to the acids.

Current research is focused on the investigation of the photophysical characteristics of the phenoxyderivatives of triazolopyrimidines (OH-DTPs) bearing OH-functionality in the different positions of the molecule and studies of their response to the different acids and bases as external stimuli.

Hydroxy group is very attractive from a biological point of view. Numerous biocompounds include $\mathrm{OH}-$ functionalities in their structure (alkaloids, flavonoids, isoflavonoids, terpenoids, lipids, fatty acids, amino acids etc.) $[9,10]$. Bioluminescent molecule (luciferin) and different synthetic fluorophores bearing this substituent are well known [11-19]. Introduction of hydroxy groups usually causes the changes in electronic properties owing to its strong electron-donating nature and increases the intramolecular charge transfer (ICT) effect. Moreover, hydroxy 
group can strengthen the fluorophore participation in intra- and intermolecular noncovalent bonding to enhance the molecule rigidity and take part in self-organization. Finally, hydroxy groups [20] can be transformed into the salts, which are more soluble in water. Deprotonation of hydroxy group may influence the optical properties of the resulted salts.

\section{Experimental}

UV-Vis absorption spectra were recorded on a PerkinElmer Lambda 35 UV-Vis spectrophotometer (Shelton, CT USA). Fluorescence of the sample solutions was measured using a Hitachi F-70oo spectrophotometer (Tokyo, Japan). The excitation wavelength was at the absorption maxima. Atmospheric oxygen contained in solutions was not removed. Concentration of the compounds in the solution was $5.0 \cdot 10^{-5} \mathrm{M}$ and $5 \cdot 0 \cdot 10^{-6} \mathrm{M}$ for absorption and fluorescence measurements, respectively. The relative fluorescence quantum yields (QY) were determined using quinine sulfate in $0.1 \mathrm{M} \mathrm{H}_{2} \mathrm{SO}_{4}$ as a standard (QY = 0.546). Absolute quantum yield study was performed on Horiba FlouroMax 4 Spectrofluorometer (Kyoto, Japan) with Quanta- $\phi$ integrating sphere using FluorEssence 3.5 Software.

DTPs 1a-e, 2a-c and 3a-b were prepared according to procedures reported in the literature and their spectral characteristics were identical to the published data $[8,21]$.

\section{Results and Discussion}

2-(Hydroxyphenyl)-4-(4-trifluoromethylphenyl)-2,4-5 $\mathrm{H}$ -

$[1,2,3]$ triazolo $[4,5-d]$ pyrimidines (HO-DTPs) 2a-c and 3a-b were prepared via the demethylation of their methoxycongeners by using $\mathrm{BBr}_{3}$ solution in dichloromethane with special temperature profile regime (Scheme 1).

The spectral and analytical data of DTPs 2a-d and 3a, b were consistent with the published data [21]. Absorption and emission spectra recorded earlier showed insignificant difference in long wavelength maxima positions compared to the corresponding MeO-DTPs derivatives. At the same time HO-DTPs quantum yields (QY) of were in 6-40fold lower. Only HO-DTP 2 a demonstrated good QY compared to QY of DTP 1a in DMSO (40.1\% and $39.4 \%$, respectively) (Table 1). However, in the other solvents QYs of HO-DTP 2 a decreased significantly.

The preliminary investigation of the influence of the different acids and bases on the optical properties of the HO-DTP solutions in DMSO showed the absence of any effect. Therefore, we included only trifluoroacetic acid (TFA) into further consideration. Absorption and emission spectral data of the HO-DTPs $\mathbf{2 a - d}$ and $\mathbf{3 a} \mathbf{3} \mathbf{b}$ in the presence of different organic bases, alkalis and TFA are collected in Table 1 . Analysis of the Table 1 data showed that addition of the $\mathrm{KOH}$ ( $5 \%$ water solution) caused red shift of the absorption and emission maxima by 20-121 and 22$169 \mathrm{~nm}$, respectively, and for the most HO-DTP QYs were decreased. Addition of TFA did not affect the photophysical properties for the most compounds, except the DTP $\mathbf{2 b}$. In this case, the maxima of absorption and emission bands were redshifted for $30 \mathrm{~nm}$ and $11 \mathrm{~nm}$ accordingly. Unlike most acids, the addition of $\mathrm{KOH}$ solution caused dramatic changes in the photophysical properties of DTPs 2a-d, 3a,b. Thus, in fluorescence spectra a strong bathochromic shift of the emission maxima of 57-169 nm was observed<smiles>[R]Nc1nc(=O)n(-c2ccc([R])cc2)c2nn(-c3ccc(O)cc3)cc12</smiles>

Scheme 1 Synthesis of OH-DTPs 2a-d and 3a,b 
Table 1 Absorption and emission data for DTPs 2a-d, 3a,b solution $\left(c=5 \cdot 10^{-6} \mathrm{M}\right)$ in DMSO before and after the addition of 500 eq. of TFA or $\mathrm{KOH}$ ( $5 \%$ solution in water)

\begin{tabular}{|c|c|c|c|c|c|c|}
\hline Entry & Compound & Acid/Base & $\lambda_{\mathrm{abs}}, \mathrm{nm}$ & $\lambda_{\mathrm{em}}, \mathrm{nm}$ & $\mathrm{SS}, \mathrm{nm} / \mathrm{cm}^{-1}$ & QY, \% \\
\hline 1 & & - & 347 & 441 & $94 / 6143$ & 40.1 \\
\hline 2 & $2 a$ & TFA & 347 & 441 & $94 / 6143$ & 18.2 \\
\hline 3 & & $\mathrm{KOH}$ & 375 & 498 & $123 / 6586$ & 19.8 \\
\hline 4 & & - & 296 & 419 & $123 / 9917$ & 0.4 \\
\hline 5 & $2 \mathbf{b}$ & TFA & 326 & 408 & $82 / 6165$ & 0.6 \\
\hline 6 & & $\mathrm{KOH}$ & 364 & 503 & $139 / 7592$ & 7.5 \\
\hline 7 & & - & 340 & 416 & $76 / 6903$ & 0.5 \\
\hline 8 & $2 c$ & TFA & 331 & 414 & $83 / 6057$ & 0.8 \\
\hline 9 & & $\mathrm{KOH}$ & 360 & 500 & $140 / 7778$ & 5.9 \\
\hline 10 & & - & 356 & 472 & $116 / 6903$ & 1.1 \\
\hline 11 & 2d & TFA & 360 & 450 & $90 / 5556$ & 0.6 \\
\hline 12 & & $\mathrm{KOH}$ & 418 & 530 & $110 / 5056$ & 0.6 \\
\hline 13 & & - & 347 & 432 & $85 / 5670$ & 0.6 \\
\hline 14 & 3a & TFA & 347 & 420 & $74 / 5092$ & 0.6 \\
\hline 15 & & $\mathrm{KOH}$ & 423 & 500 & $77 / 3641$ & - \\
\hline 16 & & - & 341 & 416 & $75 / 5287$ & 0.3 \\
\hline 17 & $\mathbf{3 b}$ & TFA & 339 & 404 & $65 / 4746$ & 0.2 \\
\hline 18 & & $\mathrm{KOH}$ & 492 & 585 & $93 / 3231$ & - \\
\hline
\end{tabular}

along with the QY decrease for the most DTPs, with the exception for $\mathbf{2 b}$ and $\mathbf{2 c}$. DTPs $\mathbf{2 b}$ and $\mathbf{2 c}$, bearing hydroxy group at ortho- and meta-position of aromatic ring $\mathrm{A}$ showed the large increase of their QYs from 0.4 and $0.5 \%$ (in pure DMSO) to 7.5 and $5.9 \%$, respectively. Thus, hydroxyl DTP derivatives display different sensitivity to the alkali media depending on their structure.

These results inspired us to check the influence of the set of bases on the photophysical properties of DTP $\mathbf{2 b}$ (Fig. 1) under the same conditions. These data indicated a significant effect of bases on both the absorption and the emission of DTP $\mathbf{2 b}$. In all the cases, the absorption and emission maxima were redshifted in range 68-110 $\mathrm{nm}$ and 19-62 nm, respectively. The effect of TEA stimulus deviates from common trend and consists in slight blue shifting $(4 \mathrm{~nm})$ (Fig. 1). The most effective were alkalis $(\mathrm{NaOH}$ and $\mathrm{KOH}$ ), whose addition to the HO-DTPs $\mathbf{2 b}$ solution in
DMSO led to large redshifts (61-62 nm) and substantial (in 9-10 folds) increasing of emission intensity. Visual emission change from colorless to green was observed for the DTPs $2 \mathbf{b}$ solution after the addition of 50 eq of $\mathrm{NaOH}$ solution, which further developed to bright green-yellow emission (Fig. 2).

Further, we studied changes in absorption and emission spectra of HO-DTP $2 \mathrm{c}$ induced by $\mathrm{NaOH}$ (Fig. 3). HO-DTP 2c was almost non-fluorescent in DMSO solution $\left(c=5 \cdot 10^{-6} \mathrm{M}\right.$ ), while the addition of $\mathrm{NaOH}$ caused an appearance of a new fluorescence maximum at $504 \mathrm{~nm}$ (Fig. 3b). The intensity of this new maximum was enhanced drastically upon the increasing amounts of $\mathrm{NaOH}$ and reached the saturation at 300 eq. Changes in absorption were also registered. In this case new maximum was located at $395 \mathrm{~nm}$ when 25 eq. of $\mathrm{NaOH}$ solution were added, and further $\mathrm{NaOH}$ addition led to increasing of this
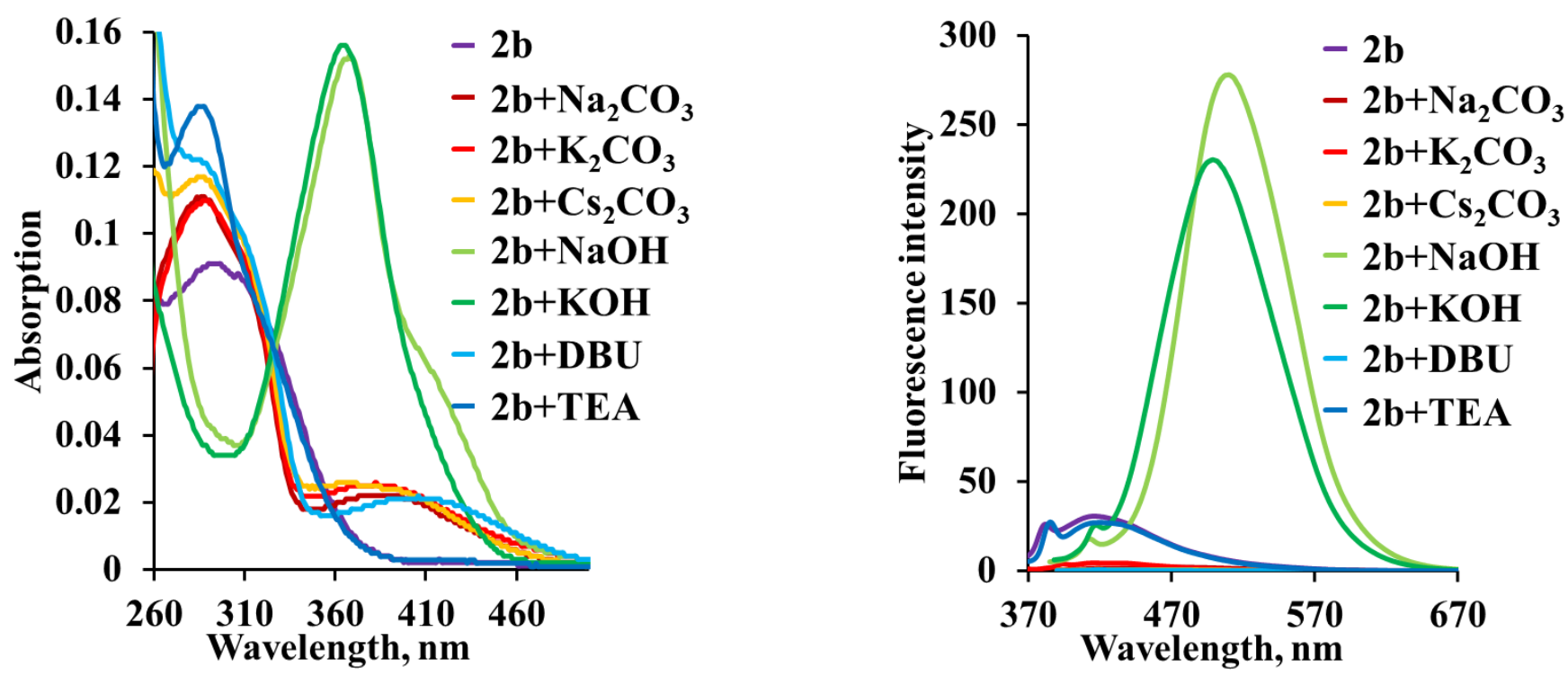

Fig. 1 UV-Vis absorption (left) and emission (right) spectra of DTP $\mathbf{2 b}$ after addition of different bases (for absorption and emission $\left.c=5 \cdot 10^{-6} \mathrm{M}\right)$ 

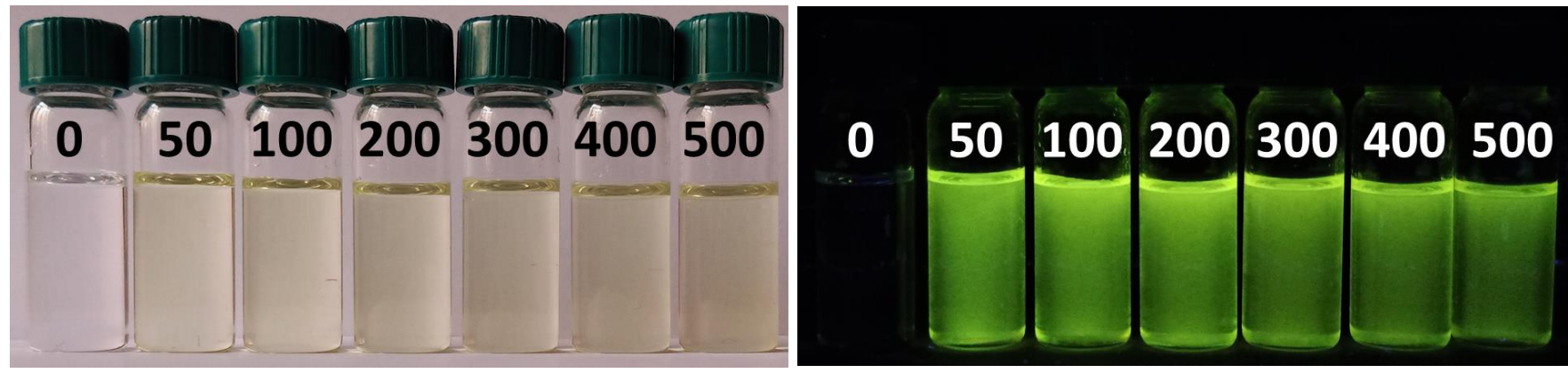

Fig. 2 The titration of HO-DTP $\mathbf{2 b}$ solution in DMSO with the $5 \% \mathrm{NaOH}$ solution under sunlight (left) and UV-irradiation (right)

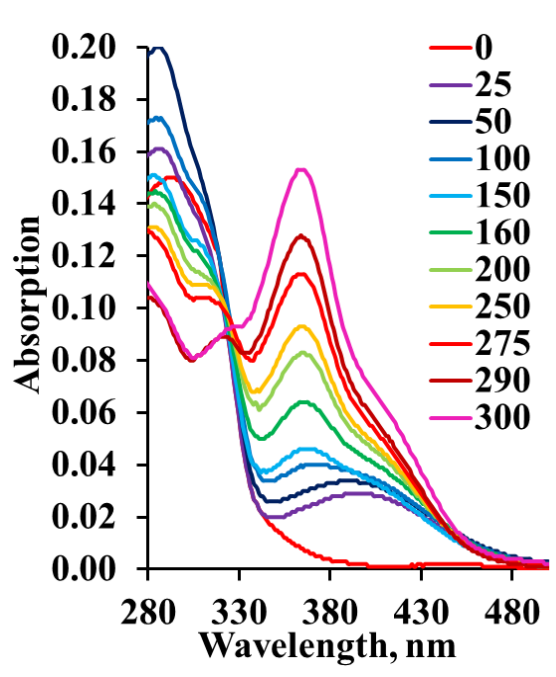

a

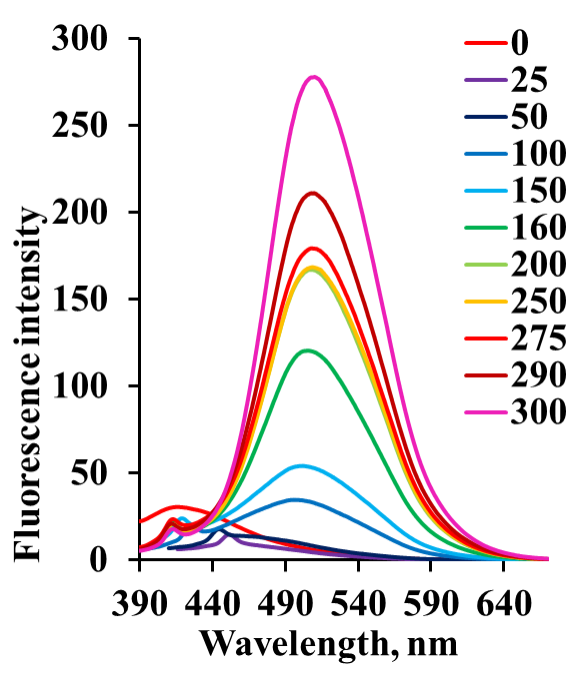

$b$

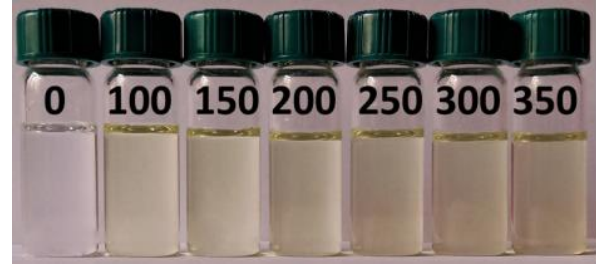

C

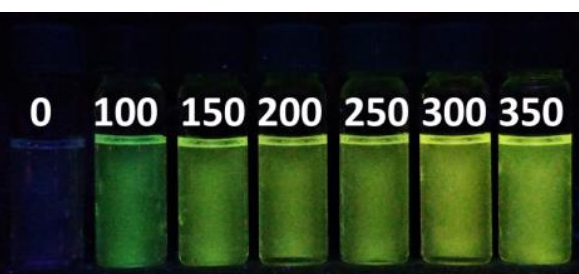

$d$

Fig. 3 Absorption (a) and emission (b) of DTP 2c solution in DMSO with addition of different eq. of NaOH. Photos of the solutions of 2c in DMSO with increasing amount of $\mathrm{NaOH}$ under sunlight (c) and UV-irradiation (d).

maximum intensity and simultaneous appearance of the new maximum at $368 \mathrm{~nm}$. Isosbestic points in absorption spectra emerged at 325 and $425 \mathrm{~nm}$ and this suggests a reactive equilibrium between these two components.

\section{Conclusions}

In conclusion, we reported the results of the investigation of the optical properties of hydroxy substituted DTPs in the presence of different acids or bases. Hydroxy-DTPs demonstrated a pronounced fluorescence response in alkaline environment, which resulted in strong bathochromic shift of the absorption and emission maxima along with enhancement of the intensity of the fluorescence. However, they did not display any noticeable perceptible response to organic or mineral acids. Thus, introducing of the $\mathrm{OH}$ group onto the dihydro-1,2,3-triazole structure led to the sharp change in response of these compounds to basic/alkali stimuli. These results together with our recent ones demonstrated that acid/base sensitivity of the DTPs can be adjusted by varying the substituents and functionalities attached to the dihydropyrimidine core.

\section{Acknowledgments}

The work was carried out with the financial support of the Russian Foundation for Basic Research (Grant 19-3390184).

\section{References}

1. Qian J, Zhang Y, Liu X, Xia J. Carbazole and fluorene polyaniline derivatives: Synthesis, properties and application as multiple stimuli-responsive fluorescent chemosensor. Talanta. 2019;204:592-601.

doi:10.1016/j.talanta.2019.06.038

2. Valeur B, Berberan-Santos MN. Molecular Fluorescence. Weinheim: Wiley VCH; 2013. 592 p.

3. Srinivasan R, Phillips TE, Bargeron CB, Carlson MA, Schemm ER, Saffarian HM. Embedded micro-sensor for monitoring pH in concrete structures. Proc SPIE. 2000;3988:40-4. doi: $10.1117 / 12.383169$

4. Basheer PAM, Grattan KTV, Sun T, Long AE, McPolin D, Xie W. Fibre optic chemical sensor systems for monitoring pH changes in concrete. Proc SPIE. 2004;5586:144-53. doi: $10.1117 / 12.601198$

5. Hecht M, Kraus W, Rurack K. A highly fluorescent pH sensing membrane for the alkaline pH range incorporating a BODIPY dye. Analyst. 2013;138(1):325-32. doi:10.1039/C2AN35860C 
6. Gotor R, Ashokkumar P, Hech M, Keil K, Rurack K. Optical pH sensor covering the range from $\mathrm{pH}$ o-14 compatible with mobile-device readout and based on a set of rationally designed indicator dyes. Anal Chem. 2017;89(16):8437-44 doi:10.1021/acs.analchem.7b01903

7. Pfeifer D, Klimart I, Borisov SM. Ultra-bright red-emitting photostable perylene bisimide dyes: new indicators for ratiometric sensing of high $\mathrm{pH}$ or carbon dioxide. Chem Eur J. 2018;24:10711-20. doi:10.1002/chem.201800867

8. Eltyshev AK, Suntsova PO, Karmatskaia KD, Taniya OS, Slepukhin PA, Benassi E, Belskaya NP. An effective and facile synthesis of new blue fluorophores on the basis of an 8azapurine core. Org Biomol Chem. 2018;16(48):9420-29. doi:10.1039/C8OB02644K

9. Li X, Peng Y, Liu H, Xu Y, Wang X, Zhang C, Ma X. Comparative studies on the interaction of nine flavonoids with trypsin. Spectrochim Acta A. 2020;238:118440. doi:10.1016/j.saa.2020.118440

10. Yao J, He Y, Su N, Bharath SR, Tao Y, Jin JM, Chen W, Song H, Tang SY. Developing a highly efficient hydroxytyrosol wholecell catalyst by de-bottlenecking rate-limiting steps. Nat Commun. 2020;11:1515. doi:10.1038/s41467-020-14918-5

11. Jadhav AS, Carreira-Blanco C, Fernández B, González Fernández S, Malkhede DD, Mosquera MM, Ríos Rodríguez MC, Rodríguez-Prieto F. Firefly luciferin precursor 2-cyano6-hydroxybenzothiazole: Fluorescence à la carte controlled by solvent and acidity. Dyes Pigm. 2020;177:108285. doi:10.1016/j.dyepig.2020.108285

12. Aysha TS, El-Sedik MS, Mohamed MBI, Gaballah ST, Kamel MM. Dyes Pigm 2019;170:107549. doi:10.1016/j.dyepig.2019.107549

13. Zhang J, Lia J, Chen B, Kan J, Jiang T, Zhang W, Yue J, Zhou J. An off-on fluorescent probe for real-time sensing the fluctuations of intracellular $\mathrm{pH}$ values in biological processes. Dyes Pigm. 2019;170:107620. doi:10.1016/j.dyepig.2019.107620
14. Bondar K, Bokan M, Gellerman G, Patsenker LD. Water-soluble 4-hydroxystyryl and 4-hydroxyphenylbutadienyls dyes with switchable fluorescence. Dyes Pigm. 2020;172:107801. doi:10.1016/j.dyepig.2019.107801

15. Seo MH, Han J, Jin Z, Lee DW, Park HS, Kim HS Controlled and Oriented Immobilization of Protein by SiteSpecific Incorporation of Unnatural Amino Acid. Anal Chem. 2011;83(8):2841-45. doi:10.1021/ac103334b

16. Eichelbaum K, Winter M, Berriel Diaz M, Herzig S, Krijgsveld J. Selective enrichment of newly synthesized proteins for quantitative secretome analysis. Nat Biotechnol. 2012;30:984-90. doi:10.1038/nbt.2356

17. Raliski BK, Howard CA, Young DD. Site-Specific Protein Immobilization Using Unnatural Amino Acids. Bioconjugate Chem. 2014;25:1916-20. doi:10.1021/bc500443h

18. Hutchins BM, Kazane SA, Staflin K, Forsyth JS, FeldingHabermann B, Schultz PG, Smider VV. Site-Specific Coupling and Sterically Controlled Formation of Multimeric Antibody Fab Fragments with Unnatural Amino Acids. J Mol Biol. 2011;406:595-603. doi:10.1016/j.jmb.2011.01.011

19. Young DD, Jockush S, Turro NJ, Schultz PG. Synthetase polyspecificity as a tool to modulate protein function. Bioorg. Med Chem Lett. 2011;21:7502-04. doi:10.1016/j.bmcl.2011.09.108

20. Gorduk S. Highly soluble HOPEMP-functionalized phthalocyanines for photodynamic activity: Photophysical, photochemical and aggregation properties. J Mol Struct. 2020;1217:128478. doi:10.1016/j.molstruc.2020.128478

21. Eltyshev AK, Minin AS, Smoliuk LT, Benassi E, Belskaya NP. 2-Aryl-2,4-dihydro-5H-[1,2,3]triazolo[4,5-d]pyrimidin-5-ones as a New Platform for the Design and Synthesis of Biosensors and Chemosensors. Eur J Org Chem. 2020;2020:316-29. doi:10.1002/ejoc.201901582 